\title{
I disturbi del comportamento alimentare (DCA): riflessioni e problematiche
}

\author{
Emilio Franzoni ', Alessandro Pellicciari ', Leonardo Sacrato ', Paola Gualandi ', Luisa lero ', \\ Ilaria Cecconi ${ }^{1}$ \\ 1 Unità Operativa Neuropsichiatria Infantile e Centro a Valenza Regionale per i Disturbi del Comportamento Alimentare in Età Evolutiva \\ "Dott.ssa Anna Andreoli” Azienda Ospedaliero Universitaria Sant’Orsola-Malpighi, Bologna
}

\begin{abstract}
Eating disorders (ED) are an important cause of physical and psychosocial morbidity in adolescents and young adults, characterized by aberrant patterns of eating behavior and weight regulation and by disturbances in attitudes towards weight and perception of body shape. This article, through the description of a clinical case, focuses on the complexities of clinical management and on the importance of an integrated approach.
\end{abstract}

Keywords: eating disorders, adolescents, clinical management

Eating disorders (ED): problems and considerations

Pratica Medica \& Aspetti Legali 2009; 3(3): 117-123

\section{INTRODUZIONE}

«Ho paura della solitudine, di far soffrire gli altri, della depressione e di mangiare tanto solo per sfogarmi, aumentando di peso a dismisura... Ho paura delle responsabilità della vita, la voglia di vivere mi spaventa, ho paura delle emozioni, ho paura di capire cosa provo veramente. Ho bisogno della malattia, ho bisogno di esserne dentro, ma ho paura che - continuando a comportarmi come voglio - mi posizionino il sondino, e rivedermi ancora più grassa, e rivedere le persone che frequentavo un tempo, e sentire le loro opinioni. Ho paura di peggiorare cosi tanto da uccidermi».

Le parole di S., una ragazza in età evolutiva ricoverata nel nostro centro per i disturbi alimentari, sono una ruvida testimonianza della estrema complessità dei disturbi del comportamento alimentare (DCA), uno spettro di condizioni che ha radici profonde in situazioni biologiche, psicologiche e sociali. Talmente complesse ne sono origini e fattezze, che si è scelto nel tempo di abbandonare l'idea di disturbo mentale, definendole piuttosto come patologie dello sviluppo, che si distribuiscono cioè lungo un continuum assumendo caratteristiche differenti nelle diverse fasce di età.

\section{DESCRIZIONE DEL CASO}

S. ha 16 anni quando viene ricoverata presso la nostra struttura per aver raggiunto un Body Mass In$\operatorname{dex}(\mathrm{BMI})$ di 13,4 (valori normali: 18,5-24). Silenziosa, rigida nella postura e sempre sorridente, non si relaziona né prende mai la parola in modo autonomo. Rimane costantemente in presenza della madre, la quale sovente risponde alle domande al posto suo. Il padre soffre dal 2003 di una grave forma di depressione, aggravata da aspetti deliranti di tipo persecutorio e manie di avvelenamento. Dal 2003 al 2005 il padre subisce tre ricoveri in TSO (trattamento sanitario obbligatorio). Durante l'ultimo ricovero tenta il suicidio. La signora riporta che la figlia ha sofferto di problemi della sfe- 
ra alimentare fin dai primi mesi di vita: la piccola, all'età di due mesi, viene ricoverata in ospedale per ipotermia, rifiuto ad alimentarsi, ed eritema cutaneo. Nonostante ciò la signora la allatta per quasi 2 anni (cospargendosi i capezzoli di amuchina per terminare l'allattamento).

Vengono riferite difficoltà nell'acquisizione del linguaggio: la bambina non comunica se non attraverso dei suoni inarticolati che capisce solo la madre; è particolare come il resto della famiglia venga esclusa da questi scambi comunicativi che riguardano esclusivamente madre-figlia; solo a 4 anni la piccola viene portata da una logopedista, ma la situazione migliora molto lentamente. Quando la piccola ha 10 mesi la madre inizia un lavoro part-time: S. piange disperatamente al momento del distacco.

In questo periodo cominciano gravi problemi relativi alla stitichezza: la bimba non evacua spontaneamente e deve essere continuamente stimolata, problema che persiste ancora oggi. Ad ogni tappa scolastica vengono riferite difficoltà nell'inserimento, con scarsa relazione con il gruppo dei pari e ansia da separazione.

All'età di 13 anni compare il menarca e, sempre in questo periodo, il padre viene ricoverato per il primo TSO; cominciano i problemi di coppia fra i genitori che decidono di "fare i separati in casa"; sempre in questo periodo la signora intraprende una relazione amorosa con un altro uomo di nascosto dalla famiglia. Solo a gennaio del 2006 i genitori vanno a vivere in due case separate e dopo qualche mese si manifestano i problemi alimentari di tipo restrittivo della figlia minore. A 16 anni S. viene considerata ancora come una bimba piccola: non può mai essere lasciata sola, non le viene comunicato mai nulla, non partecipa alle decisioni familiari, le relazioni amicali vengono promosse solo in presenza della madre.

Al momento del ricovero S. non si presenta autonoma in nessuna delle sue necessità. Non parla né si relaziona con nessuno, non mangia, non beve e la madre la sostituisce in tutto (parla al suo posto se le si rivolge una domanda diretta, la veste e la cura come una bambina piccola). Léquipe si trova costretta a posizionarle un SNG (sondino nasogastrico) a causa del fermo rifiuto di S. di bere e mangiare, e la ragazza per diverso tempo sembra sentirsi emotivamente sollevata dal non doversi alimentare autonomamente.

Durante le attività di gruppo rimane in disparte, è taciturna e sempre poco partecipe. Ai colloqui individuali parla solo della difficoltà con il cibo.

La relazione fra i curanti e la mamma di S. inizia con molta difficoltà: la signora spesso boicotta gli interventi dell'équipe; tutto ciò che riguarda la figlia deve passare attraverso lei (terapia farmacologia, scuola, test psicologici, attività riabilitative ecc.). L'équipe percepisce la difficoltà di porre la diagnosi: lo spazio soggettivo di S. è coperto e soffocato dalla madre e diventa quasi impossibile valutare l'entità del disturbo della ragazza. I medici decidono quindi un allontanamento della madre dalla struttura, mantenendo tuttavia un ulteriore momento settimanale di colloquio per lavorare sull'alleanza terapeutica. In questi termini la signora riesce a prendere le distanze dalla figlia e a ripresentarsi solo la sera e in momenti strutturati. È circa in questo periodo che S., lentamente, riprende ad alimentarsi in modo autonomo. Le frequenti crisi di rabbia della ragazza diminuiscono e si concentrano nei pochi momenti in cui la madre è presente. In un episodio particolarmente significativo, S., successivamente a un litigio con la madre, tenta di buttarsi dalla finestra. Questo episodio viene risolto dalla madre stessa, coadiuvata dal tempestivo intervento degli infermieri.

Nonostante ciò i colloqui individuali diventano più vivaci: emerge la rabbia nei confronti del padre, la paura di avere la sua stessa malattia, la paura di perdere il disturbo alimentare e soprattutto la sua paura di crescere. S. dice: «se ricomincio a mangiare, divento grande e perdo l'amore di mia mamma; c'è una parte di me che vorrebbe guarire e un'altra parte che vuole rimanere bambina per non avere responsabilità, per non affrontare gli altri».

Emerge in modo evidente la paura di essere dimessa dall'ospedale: tornare a scuola e alla vita di tutti i giorni la terrorizza; sente di non essere in grado di affrontare tutto ciò. Afferma quindi: «Non voglio essere dimessa, qui mi sento sicura, protetta. Essere dimessa significa dire a tutti di essere guarita». Al momento della dimissione si programma un percorso terapeutico in collaborazione con una struttura che si occupa di psichiatria per l'età evolutiva, che possa coadiuvarci nel trattamento della ragazza. Viene impostata una psicoterapia individuale settimanale da associarsi al trattamento farmacologico. Vista la buona relazione terapeutica intrapresa, decidiamo di far proseguire il trattamento psicoterapeutico alla madre, iniziato durante il ricovero. Si propone inoltre un graduale rientro a scuola.

Dopo quattro mesi S. viene nuovamente ricoverata presso la nostra struttura in accordo con l'équipe di psichiatria dell'età evolutiva: viene riferito un deterioramento del rapporto con la madre, che si manifesta attraverso episodi di etero-aggressività piuttosto intensi, isolamento, e una concreta difficoltà nell'inserimento scolastico. Inoltre la paziente rifiuta nuovamente di alimentarsi. Notiamo già come le frequenti crisi violente subiscano una forte riduzione alla ripresa di una alimentazione restrittiva. Sembra che il disturbo alimentare venga utilizzato in modo difensivo, quasi a contenere una patologia ben più grave e di difficile gestione.

Al secondo ricovero $S$. si caratterizza per una importante sintomatologia ossessivo-compulsiva. È sempre attenta ai suoi capelli, alla direzione nello 
spazio dei suoi oggetti, agli orari in cui assumere la terapia farmacologica. Appare padrona del reparto, sembra che per lei l'ospedale funga da casa. Pare il luogo in cui ha trovato rifugio e da cui non vuole più uscire. Si relaziona con gli altri e si preoccupa delle difficoltà altrui, anche se sono presenti importanti sentimenti di esclusione. Le viene posizionato anche questa volta un SNG, che la paziente accoglie con tranquillità, poiché le dà la garanzia di essere effettivamente anoressica e la mette così al riparo dalle proprie angosce. Durante il secondo ricovero $\mathrm{S}$. attraversa varie crisi, causate dal confronto con gli altri e dal ritorno del ciclo mestruale. In questo periodo vengono riportate tematiche costanti, quali la paura di non farcela nelle diverse situazioni, l'angoscia di crescere e di diventare grande.

Rispetto al rapporto con la madre S. prende consapevolezza della grande ambivalenza che prova nei suoi confronti e lentamente mette in atto alcuni deboli tentativi di autonomia; comincia a telefonarle meno, ad esprimere delle critiche nei suoi confronti, a nasconderle alcuni dettagli inerenti il suo piano terapeutico. Inoltre sembra manifestare un grande e autentico piacere durante gli incontri di pet therapy. S. manifesta il desiderio di poter possedere un cane del quale potersi prendere cura in modo autonomo. Programmiamo quindi insieme ai genitori la possibilità di poterne acquistare uno una volta dimessa, e, con l'aiuto del nostro terapeuta, la possibilità di cominciare ad addestrarlo.

Ma la radicata e pervasiva dipendenza dalla madre pare in parte spostarsi sulla struttura ospedaliera: la paziente si affida pienamente all'équipe che diventa per lei un punto di riferimento, dal quale teme costantemente un abbandono. Cominciano i permessi a casa che la ragazza affronta con una profonda angoscia: durante un permesso, in occasione di una crisi, vive un episodio di depersonalizzazione.

Viene quindi attivato un progetto integrato: da un lato si intraprende un percorso ambulatoriale presso la nostra struttura, dall'altro si attiva un regime di semiresidenza in un centro per adolescenti presso l'unità di psichiatria dell'età evolutiva che viene faticosamente accettato dalla ragazza.

Durante la degenza presso la semiresidenza S. conosce ed entra in competizione con un'altra paziente: sebbene quando sotto osservazione la sua sintomatologia appaia in remissione, nei momenti trascorsi a casa la ragazza accentua l'iperattività, le condotte ossessive divengono più pervasive $e$ compaiono episodi di auto- ed etero-aggressività. Quando, nel tentativo di incrementare l'attività, si getta a correre in strada nel traffico cittadino appare chiaro che i suoi comportamenti la pongono in serio pericolo. Tali atteggiamenti non vengono riferiti agli psichiatri che l'hanno in cura, ma soltanto al nostro Centro. Diviene allora fondamenta- le il lavoro di rete: da un lato l'informazione viene passata all'unità di psichiatria, dall'altro alla famiglia stessa, affinché maturi la consapevolezza del reale pericolo che $\mathrm{S}$. corre. L'unità di psichiatria predispone un ricovero, evenienza che viene però rifiutata dalla famiglia: si rende quindi necessario il ricorso a un trattamento sanitario obbligatorio. L'introduzione di una nuova terapia farmacologica e l'intensificarsi degli incontri portano $S$. e la sua famiglia ad accettare il trasferimento presso una clinica privata. Alla dimissione il pericolo immediato appare scongiurato, sebbene permanga radicato in S. il disturbo alimentare.

Attualmente S. ha compiuto 18 anni e continua ad essere seguita presso il nostro Centro e presso la semiresidenza di psichiatria. Il percorso riabilitativo e psicoeducazionale di S. appare ancora lungo, ma il lavoro integrato, con il quotidiano scambio di informazioni tra i due Centri, sembra in grado di contenerla nei suoi momenti di scompenso.

\section{GLI ASPETTI CLINICI}

\section{CLASSIFICAZIONE E PREVALENZA}

Il principale sistema nosografico categoriale di inquadramento delle patologie psichiatriche, il DSM-IV, distingue i DCA in anoressia nervosa (AN), bulimia nervosa (BN) e disturbi del comportamento alimentare non altrimenti specificati (DCANAS) [1]. In Tabella I e in Tabella II sono riportati i criteri del DSM-IV per anoressia e bulimia nervosa, rispettivamente.

I DCA sono condizioni estremamente frequenti: è stimata una prevalenza dello $0,3 \%$ in giovani donne per quanto riguarda la $\mathrm{AN}$, dell' $1 \%$ e dello $0,1 \%$ rispettivamente in ragazze e ragazzi per la $\mathrm{BN}$, mentre la prevalenza dei DCANAS sembra essere del $6 \%$. Si stima che il disturbo affligga per il 90-95\% il sesso femminile, ma si deve registrare l'incremento della prevalenza nel sesso maschile negli ultimi anni [2]. Sebbene l'esordio sia rico-

II rifiuto di mantenere il peso corporeo al di sopra o al peso minimo normale per l'età e statura

- L'intensa paura di acquistare peso o di diventare grassi, anche quando si è sottopeso

- L'alterazione del modo in cui il soggetto vive il peso o la forma del corpo, o l'eccessiva influenza del peso e della forma del corpo sui livelli di autostima, o il rifiuto di ammettere la gravità della attuale condizione di sottopeso

- Nelle femmine dopo il menarca, I'amenorrea, cioè l'assenza di almeno tre cicli mestruali consecutivi

Tabella I. Criteri per porre diagnosi di anoressia nervosa [1] 


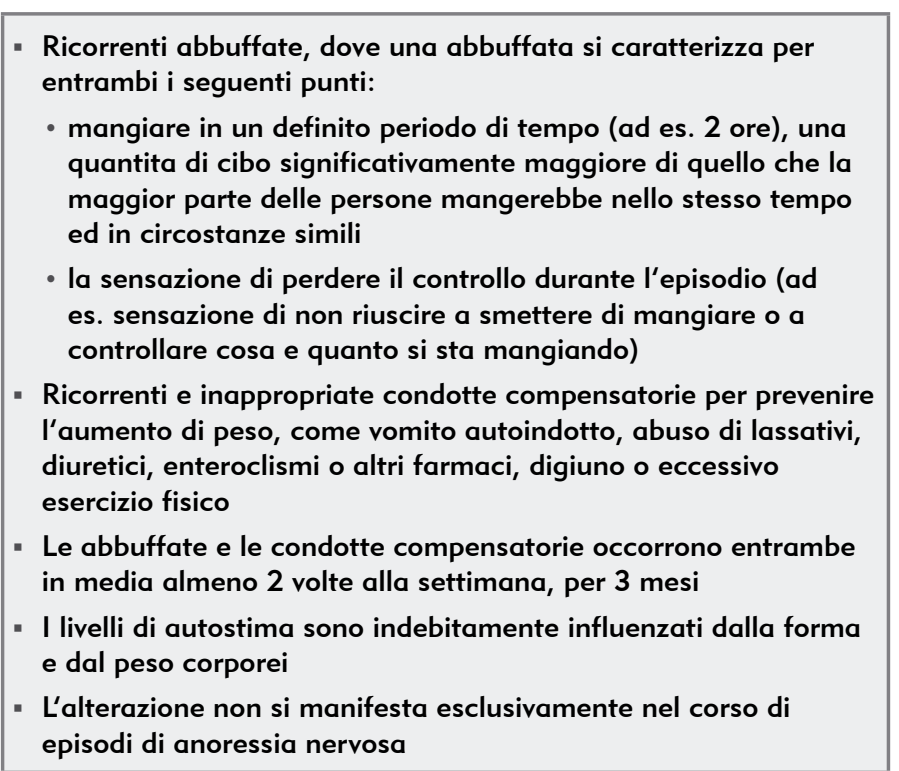

Tabella II. Criteri per porre diagnosi di bulimia nervosa [1]

\begin{tabular}{|ll|}
\hline $\begin{array}{l}\text { Anoressia } \\
\text { nervosa }\end{array}$ & $\begin{array}{l}\text { Rifiuto ad alimentarsi, distorsioni cognitive e } \\
\text { preoccupazione eccessiva per il peso }\end{array}$ \\
\hline $\begin{array}{l}\text { Bulimia } \\
\text { nervosa }\end{array}$ & Abbuffate e condotte eliminatorie senza controllo \\
\hline $\begin{array}{l}\text { Disturbo } \\
\text { emotivo di } \\
\text { rifiuto del cibo }\end{array}$ & $\begin{array}{l}\text { Rifiuto del cibo associato a un disturbo dell'umore } \\
\text { senza distorsioni cognitive e preoccupazione per } \\
\text { il peso }\end{array}$ \\
\hline $\begin{array}{l}\text { Alimentazione } \\
\text { selettiva }\end{array}$ & $\begin{array}{l}\text { Forte selettività e rifiuto di nuovi alimenti in } \\
\text { assenza di distorsioni cognitive e aspetti fobici }\end{array}$ \\
\hline $\begin{array}{l}\text { Disfagia } \\
\text { funzionale }\end{array}$ & $\begin{array}{l}\text { Evitamento del cibo associato alla paura del } \\
\text { soffocamento o vomito senza distorsioni cognitive }\end{array}$ \\
\hline $\begin{array}{l}\text { Rifiuto } \\
\text { pervasivo }\end{array}$ & Rifiuto totale di alimentarsi e prendersi cura di sé \\
\hline
\end{tabular}

Tabella III. Classificazione elaborata dal Workgroup for Classification for Eating Disorders in Children and Adolescent (WCEDCA, 2007) [3]

nosciuto nell'età adolescenziale, recenti segnalazioni pongono all'attenzione l'abbassamento della soglia d'insorgenza di tutti i DCA, con la comparsa sempre più frequentemente del disturbo in età prepubere, dove la differenza di sesso è certamente molto meno significativa. Infatti, nella valutazione delle diverse fasce di età, un comportamento "anoressico" è già sicuramente osservabile nel corso della prima infanzia (0-3 anni) mentre un comportamento a tipo "abbuffate" è invece riscontrabile dalla terza infanzia (6-10 anni).

I disturbi alimentari nell'infanzia comprendono una varietà di problemi specifici con eziologie ed esiti diversi: in genere si stima che il $25 \%$ dei bambini con un normale sviluppo psicofisico e il $35 \%$ in bambini con una difficoltà di sviluppo possano presentare un problema alimentare.
I dati epidemologici che riguardano i disturbi gravi indicano una prevalenza del $5-10 \%$ nei primi quindici mesi di età.

Laumento di incidenza dei DCA nelle fasce d'età più precoci ha portato allo sviluppo di nuove $\mathrm{e}$ specifiche classificazioni diagnostiche (in Tabella III la classificazione elaborata dal Workgroup for Classification for Eating Disorders in Children and Adolescent) [3]. Particolarmente indicata per la prima infanzia è invece la classificazione di Irene Chatoor, che esplicita [4]:

- disturbo alimentare dell'omeostasi (esordio 0-3 mesi): il bambino fatica a raggiungere e mantenere uno stato di calma per mangiare;

- disturbo alimentare dell'attaccamento (2-8 mesi): assenza di adeguata reciprocità durante l'alimentazione con il caregiver;

- anoressia infantile (6 mesi-3 anni): rifiuto di un'adeguata quantità di cibo e essenza di interesse per lo stesso;

- avversione sensoriale al cibo: rifiuto di mangiare specifici cibi con certi odori, gusti, consistenza e colori;

- disturbo alimentare post-traumatico;

- disturbo associato a una concomitante condizione medica.

Una volta identificato il problema, diviene necessario attuare un intervento clinico, che sia incentrato sui meccanismi e sugli effetti che il sintomo ha sull'intera famiglia. È importante identificare il messaggio che il disturbo veicola, e capirne il funzionamento nell'economia dell'intero assetto familiare: dalle reazioni distruttive sull'intorno del paziente agli eventuali guadagni secondari di quest'ultimo. In età evolutiva, pertanto, non ci si può limitare alla presa in carico del singolo paziente, ma l'intero nucleo familiare dovrà essere assistito; dovranno essere inoltre considerate quelle caratteristiche uniche della fase di preadolescenza, dove coesistono aspetti caratteristici dell'infanzia e modelli di riferimento già adolescenziali, bisogno di dipendenza e spinta all'autodeterminazione, e dove lo sviluppo della capacità critica porta la ragazza a riconoscere i limiti dei propri genitori, generando così rapporti ambivalenti.

\section{ANORESSIA NERVOSA: QUADRO CLINICO E LABORATORISTICO}

Sebbene il sintomo più eclatante nella paziente con anoressia di sesso femminile sia l'amenorrea, il quadro clinico comprende:

- modificazioni fisiche come intolleranza al freddo, secchezza della cute, edemi, lipotimie, vertigini, parestesie, disturbi del sonno. Frequenti sono anche le manifestazioni ga- 
stroenterologiche: da sintomi simil-reflusso (esofagite, esofago di Barrett, dolore e bruciore epigastrico, disfagia, più tipici laddove compaiano condotte di abbuffate e compensazione) a sintomi di tipo dispeptico (distensione gastrica e addominale, ripienezza post-prandiale, sazietà precoce, più frequenti laddove prevalgano le condotte puramente restrittive). Di comune riscontro è una stipsi ostinata. Numerose sono le complicanze endocrinologiche, come ad esempio l'aumentato rischio di osteoporosi;

- modificazioni cognitive: diminuita concentrazione, povertà di giudizio critico e apatia;

- effetti psichici: isolamento sociale, labilità emotiva, ansia, depressione, irritabilità, pensieri ossessivi che possono andare dalla franca fobia (per esempio del cibo) a rituali più o meno elaborati (per esempio di contaminazione), episodi psicotici.

Gli esami di laboratorio possono rivelare anemia normocromica e normocitica, leucopenia, ipercolesterolemia, iperazotemia, ipercreatininemia, aumento degli enzimi di funzionalità epatica, ipoglicemia e ridotti livelli ormonali (particolarmente estrogeni, cortisolo e ormoni tiroidei). In caso di vomito autoindotto si può riscontrare alcalosi metabolica, mentre in caso di abuso di lassativi si rileva acidosi metabolica.

\section{LE IPOTESI DI PREVENZIONE}

Un intervento precoce può essere attuato su tre livelli, mediante:

- la promozione di una corretta alimentazione. L'alimentazione nei primi anni di vita è in grado di influenzare abitudini e stili di vita anche a lungo termine. Una corretta gestione delle fasi precoci dell'allattamento/divezzamento, caratterizzata da scambi relazionali funzionali e da una comunicazione emotivo-affettiva efficace, è il primo fattore di protezione [5];

- la corretta informazione riguardo la diffusione e la morbilità dei disturbi alimentari;

- l'intervento psicosociale, volto a rafforzare i fattori protettivi come l'educazione ai valori etici, la responsabilità individuale, lo sviluppo della capacità critica. Vanno promossi obiettivi individuali come autostima, capacità di comunicazione e gestione delle emozioni, un approccio più critico verso i modelli socioculturali che spingono alla magrezza, lo spostamento del pensiero dal corpo al Sé nella sua interezza, la riduzione del perfezionismo, il miglioramento della comunicazione intrafamiliare e lo sviluppo di strategie di coping.

\section{IL TEMPO DELLA CURA: IL CENTRO PER I DISTURBI ALIMENTARI}

Il nostro Centro è stato riconosciuto come centro a valenza regionale per la cura dei disturbi del comportamento alimentare, conforme alle indicazioni ministeriali e alle linee guida regionali che sottolineano la centralità del ruolo di un'équipe multidisciplinare comprendente: neuropsichiatra infantile, pediatra, psicologo clinico, dietista, infermiere e ausiliario. Sottolineiamo, relativamente al nostro Centro, l'importanza della figura del volontario formato attraverso appositi corsi introduttivi.

Il nostro Centro si struttura su tre ambiti: reparto, day-hospital e ambulatorio, strutture strettamente interconnesse per meglio calibrare un opportuno percorso terapeutico che non manchi di continuità.

L'accesso può provenire dal medico curante, dallo psicologo terapeuta, dalla famiglia o dal paziente stesso; infine l'invio può provenire da un altro centro o dal Pronto Soccorso in caso di acuzia.

Il percorso diagnostico comprende anamnesi, valutazione internistica e misurazione dei dati antropometrici (peso, altezza e BMI), indagini laboratoristiche (biochimiche, enzimatiche, ormonali e indici di flogosi) e indagini strumentali (elettrocardiogramma, densitometria ossea, gastroscopia, ecografia pelvica; di secondo livello sono ecocardiografia e risonanza magnetica encefalo). È inoltre necessario l'avvio di un percorso psicodiagnostico attraverso i colloqui di consultazione con la paziente e con i genitori e la somministrazione di test psicometrici e proiettivi, come ad esempio MMPI (Minnesota Multiphasic Personality Inventory), EDI-3 (Eating Disorder Inventory), SAFA (scale psichiatriche di autosomministrazione per fanciulli e adolescenti), test della figura umana di Machover, disegno della famiglia e test di Rorschach.

Pertanto, in base alla diagnosi, si forniscono informazioni per:

- una psicoterapia individuale, familiare, di coppia;

- un trattamento di supporto;

- una terapia farmacologica.

Attraverso la discussione dei dati raccolti in équipe si giunge alla sintesi diagnostica, l'inquadramento viene restituito alla paziente e ai suoi genitori e viene fornita un'indicazione del progetto individuale di trattamento e riabilitazione scelto tra varie opportunità: Centro, Servizi territoriali o Strutture private.

Le modalità di intervento in ambulatorio includono:

- valutazione clinica, psicologica e dietologica;

- controlli internistici e dietologici;

- periodici colloqui psicologici con la famiglia; 


\begin{tabular}{|ll|}
\hline $\begin{array}{l}\text { Art. 32 della Costituzione } \\
\text { italiana }\end{array}$ & $\begin{array}{l}\text { «La Repubblica tutela la salute come fondamentale diritto dell'individuo e } \\
\text { interesse della collettività, e garantisce cure gratuite agli indigenti. Nessuno } \\
\text { può essere obbligato a un determinato trattamento sanitario se non per } \\
\text { disposizione di legge» }\end{array}$ \\
\hline $\begin{array}{l}\text { Art. 33, n. } 833 \text { del legge } \\
\text { 23 dicembre 1978 "Norme } \\
\text { per gli accertamenti e i } \\
\text { trattamenti sanitari volontari } \\
\text { e obbligatori" }\end{array}$ & $\begin{array}{l}\text { "Gli accertamenti e i trattamenti sono di norma Volontari. [...] Possono } \\
\text { essere disposti dall'autorità sanitaria accertamenti e trattamenti sanitari } \\
\text { obbligatori, secondo l'articolo 32 della Costituzione, nel rispetto della dignità } \\
\text { della persona e dei diritti civili e politici, compreso per quanto possibile il } \\
\text { diritto alla libera scelta del medico e del luogo di cura» }\end{array}$ \\
\hline $\begin{array}{l}\text { Art. 34, legge n. 833 } \\
\text { del 23 dicembre 1978 } \\
\text { "Accertamenti e trattamenti } \\
\text { sanitari volontari e } \\
\text { obbligatori" }\end{array}$ & $\begin{array}{l}\text { "La legge regionale [...] disciplina l'istituzione di servizi a struttura } \\
\text { dipartimentale che svolgono funzioni preventive, curative e riabilitative } \\
\text { relative alla salute mentale. Le misure di cui al secondo comma dell'articolo } \\
\text { precedente possono essere disposte nei confronti di persone affette da } \\
\text { malattia mentale. [...] Il trattamento sanitario obbligatorio per malattia } \\
\text { mentale può prevedere che le cure vengano prestate in condizioni di degenza } \\
\text { ospedaliera solo se esistano alterazioni psichiche tali da richiedere urgenti } \\
\text { interventi terapeutici, se gli stessi non vengano accettati dall'infermo e se non } \\
\text { vi siano le condizioni e le circostanze che consentano di adottare tempestive } \\
\text { ed idonee misure sanitarie extraospedaliere» }\end{array}$ \\
\hline
\end{tabular}

Tabella IV. Leggi che regolano il trattamento sanitario obbligatorio

- colloqui motivazionali;

- gruppi di auto-aiuto e gruppi di ascolto per i genitori.

In reparto, che vede come suo momento fondamentale la degenza, si eseguono esami e protocolli nutrizionali con pasti assistiti, colloqui psicologici bisettimanali con la paziente e settimanali con la famiglia, psicoterapia di gruppo dei genitori delle pazienti ricoverate, attività riabilitative (teatro, laboratorio del legno, di espressione artistica, ecc.). Al termine della degenza, che nel nostro Centro dura mediamente due mesi, si decide l'invio a Strutture territoriali pubbliche e/o private o a strutture interne.

\section{GLI ASPETTI LEGALI}

\section{IL TRATTAMENTO SANITARIO OBBLIGATORIO NEI DCA}

Il TSO è un trattamento sanitario che prescinde nell'immediato dal consenso del paziente (o, nell'eventualità in cui si tratti di minore, come nel caso descritto, dei genitori) e che si attua in caso di dissenso alle cure o di non validità del consenso [6]. Di per sé il trattamento in assenza di consenso rappresenta una limitazione della libertà, diritto fondamentale della persona sancito dall'art. 2 della Costituzione (Tabella IV), ed è pertanto una misura eccezionale che deve avere precise limitazioni nei parametri clinici e nella durata del trattamento, deve essere sempre motivato in modo rigoroso e deve essere accompagnato dal tenta- tivo di coinvolgere comunque il paziente nel trattamento [6].

La legislazione che riguarda il TSO è definita in maniera chiara soltanto per i soggetti con alterazioni mentali. Il provvedimento viene emanato dal sindaco in presenza di due certificazioni mediche che attestino che la persona si trova in una situazione mentale alterata, tale da rendere necessari interventi terapeutici urgenti, che gli interventi precedentemente proposti sono stati rifiutati, e che non è possibile adottare misure extraospedaliere.

L'applicazione del TSO a soggetti con anoressia nervosa è controversa. Si può infatti discutere se si tratta di una patologia che mostri le caratteristiche previste dalla legge 833/78 (Tabella IV) per l'imposizione del TSO, ossia se «esistano alterazioni psichiche tali da richiedere urgenti interventi terapeutici, se gli stessi non vengano accettati dall'infermo e se non vi siano le condizioni e le circostanze che consentano di adottare tempestive ed idonee misure sanitarie extraospedaliere».

Secondo alcuni Autori nell'AN il motivo urgente dell'ospedalizzazione è costituito dalla compromissione fisica, che richiede un trattamento di carattere medico e/o la rialimentazione forzata; secondo altri la motivazione è dettata dai disturbi psichiatrici legati alla patologia: in alcune pazienti è infatti presente un deterioramento del pensiero, della percezione del giudizio, del comportamento e della capacità di rispondere alle normali necessità di sopravvivenza [7].

La decisione sull'eventualità di un trattamento sanitario obbligatorio nei DCA è talmente controversa da aver portato alla proposta di modificare la legge 180 ("Accertamenti e trattamenti sanitari volontari e obbligatori”, del 13 maggio 1978), così da poter attuare un TSO esclusivamente mirato a 
questa patologia. Sulla base delle conoscenze in essere, è difficile predire se un trattamento obbligatorio può diventare necessario per proteggere la vita e la salute della paziente. Depongono a favore della sua inevitabilità:

- un disordine pervasivo della personalità con precedenti episodi di autolesionismo;

- la presenza di una severa compromissione fisica (BMI < 13), eventualmente caratterizzata da precedenti ricoveri;

- l'impossibilità di gestione da parte del singolo o della famiglia.

Giova ricordare che il TSO è efficace soprattutto nel breve periodo, e che il rischio di mortalità a lungo termine sussiste: è pertanto essenziale organizzare uno stretto follow-up [8]. Naturalmente il medico, qualora ritenga che la patologia rappresenti un effettivo pericolo per la vita della paziente, e quindi ritenga opportuno proporre il TSO, cercherà sempre l'alleanza terapeutica con il malato, obiettivo primo della psicoterapia stessa.

\section{CONCLUSIONI}

La nostra esperienza sostiene l'efficacia di un approccio integrato e multidisciplinare al paziente. Tale modalità permette di coinvolgere e organizzare le parti della sofferenza del bambino, dell'adolescente e della sua famiglia elaborandole all'interno di un percorso terapeutico unitario e coerente.

L'eterogeneità delle manifestazioni sintomatologiche alimentari oggi conosciute impone l'esigenza di effettuare una globale gestione della realtà personale e sintomatica del paziente.

\section{CORRESPONDING AUTHOR}

Prof. Emilio Franzoni

U.O. Neuropsichiatria Infantile e Centro a valenza regionale per i disturbi del comportamento alimentare in età evolutiva "Dott. ssa Anna Andreoli", Azienda Ospedaliero Universitaria Sant’Orsola Malpighi

Via Massarenti 11 - 40100 Bologna 